\title{
Optimizing the approach to pain, agitation, and delirium in critical care
}

\author{
Jan O. Friedrich, MD • Andrew J. Baker, MD
}

Received: 10 March 2014/Accepted: 17 April 2014/Published online: 6 May 2014

(C) Canadian Anesthesiologists' Society 2014

Critically ill patients whose lungs are mechanically ventilated almost universally receive analgesia and sedation and frequently receive antipsychotic and neuromuscular blockers. These interventions are used in attempts to minimize the discomfort of invasive physiologic support and to improve patients' tolerance and experience with intensive care in general. Similar to the concept of the "triad of anesthesia" (analgesia, sedation, and muscle relaxation), the concept of the "triad of intensive care", which consists of addressing pain, agitation, and delirium, has been proposed for critically ill patients. ${ }^{1}$ The most recently published guidelines for the management of pain, agitation, and delirium in the intensive care unit (ICU) ${ }^{2}$ recommend: 1) regularly assessing levels of pain, sedation, and delirium using validated scales; 2 ) prioritizing the treatment of pain

J. O. Friedrich, MD

Departments of Critical Care and Medicine, Keenan Research Centre for Biomedical Science and Li Ka Shing Knowledge Institute of St. Michael's Hospital, Toronto, ON, Canada

J. O. Friedrich, MD

Department of Medicine and the Interdepartmental Division of Critical Care Medicine, University of Toronto, Toronto, ON, Canada

A. J. Baker, MD $(\bowtie)$

Departments of Critical Care and Anesthesia, Keenan Research Centre for Biomedical Science and Li Ka Shing Knowledge Institute of St. Michael's Hospital, Toronto, ON M5B 1W8, Canada

e-mail: bakera@smh.ca

\section{A. J. Baker, MD}

Departments of Anesthesia and Surgery, and the Interdepartmental Division of Critical Care Medicine, University of Toronto, Toronto, ON, Canada using both non-pharmacologic and pharmacologic means; 3) prioritizing non-pharmacologic means to prevent and treat delirium; and 4) using sedatives to maintain only light rather than deep levels of sedation.

This strategy aims to treat rather than to mask disease by employing sedatives only after pain and delirium have been treated. ${ }^{1}$ Although clinicians may have previously considered deeper sedation necessary to improve patientventilator synchrony (especially when ICU ventilators were less capable of synchronizing with patients' respiratory efforts) and to minimize perceived psychological trauma, recent trials have shown that the use of lighter sedation results in no worse $^{3}$ or actually decreased adverse psychological outcomes. ${ }^{4,5}$ Thus, investigators involved in this important field of research are pursuing the best way to approach this ICU triad while minimizing potential detrimental effects of the pharmacologic agents employed and optimizing important patient outcomes, such as minimizing the duration of ventilation and length of ICU stay.

In this month's issue of the Journal, Burry et al. ${ }^{6}$ report the results of a prospective cross-sectional observational study in medical and surgical ICUs. The authors recorded use of analgesics, sedation, antipsychotics, and neuromuscular blockers in consecutive patients who were initiated on invasive mechanical ventilation during a twoweek period. The patients were followed until tracheal extubation, tracheostomy, death, or a maximum of 30 days. The focus of the study was on the use of these interventions but not on their impact on duration of ventilation or length of stay. Data were collected from a convenience, though large, sample of 51 ICUs across Canada, and according to the authors, they targeted a diverse sample, including both university and community hospitals, though they presumably needed to have the ability to collect 
prospective data. Seven hundred twelve patients were enrolled from February 2008 to April 2009, five years prior to publication and prior to publication of the most recent guidelines. ${ }^{2}$ Nearly all patients $(92 \%)$ received analgesics or sedatives, primarily as continuous infusions, with opioids used more frequently ( $85 \%$ ) than benzodiazepines $(62 \%)$ and propofol used infrequently (10\%). Antipsychotics were also used in $10 \%$ of patients, almost exclusively in those whose lungs were ventilated for more than $48 \mathrm{hr}$. Neuromuscular blockers were used in $18 \%$ of patients, which perhaps is a surprisingly high proportion given that patients' lungs were ventilated for a median of only three days. On the other hand, only 58/130 patients receiving neuromuscular blocking agents received more than a single dose, so it is difficult to know whether the single doses were procedure-related or used to assist with ventilation. For patients whose lungs were ventilated seven days or longer, opioids and benzodiazepines were used equally frequently ( $92.6 \%$ vs $92.1 \%$, respectively). The use of both opioids and benzodiazepines was independently associated with longer duration of mechanical ventilation, the use of analgesia or sedation scales, and the use of physical restraints. Sedation, pain, and delirium scales were used in 53\%, 19\%, and 5\% of patients, respectively, and $25 \%$ of analgesics and/or sedatives were titrated using a protocol. Sedation protocols were more likely to be used when scales were used - this makes sense since most protocols involve titration to a scale- in patients whose lungs required longer duration of ventilation and in community ( $v s$ university) hospitals. The authors concluded that "Despite professional society recommendations, the use of continuous infusions without targeted sedation strategies, such as a protocol, was frequent."

Burry et al. ${ }^{6}$ also reported that $22 \%$ of all sedative and opioid infusions were stopped and resumed as part of a planned daily interruption strategy. Ever since the publication of a randomized controlled trial (RCT) by Kress et al. in $2000,{ }^{7}$ daily interruption of sedation has driven research in this field as a method to avoid any potential adverse effects of sedation. This landmark trial involving 128 critically ill patients randomized to daily interruption of sedation $v s$ interruption only at the discretion of clinicians resulted in a decrease in the use of sedatives and opiate analgesics, a decrease in the median days of mechanical ventilation by 2.4 days (4.9 vs 7.3, respectively; $P=0.004$ ), and a decrease in the median days of ICU stay by 3.5 days (6.4 vs 9.9, respectively; $P=0.02$ ). In this single-centre trial, sedation infusions were titrated to achieve a score of 3-4 on the Ramsay sedation scale that categorizes sedation from 0 (agitated or restless) to 6 (asleep and unresponsive to stimuli). ${ }^{8}$ This RCT was followed by a four-centre RCT involving 336 patients whose daily interruption of sedation infusions was combined with a daily spontaneous breathing trial (the Awakening and Breathing Controlled trial) ${ }^{9}$ to assess patients for tracheal extubation. The group receiving this intervention was compared with a control group that was also assessed for daily spontaneous breathing and sedation scales were employed. This trial also showed similar benefits, but unlike the original landmark trial, there were significantly more unplanned self-tracheal extubations in the daily awakening (infusion interruption) group (10\% vs $4 \%$, respectively; $P=0.03$ ).

Other research groups have had difficulty achieving reduced sedative doses and corresponding improvements in lengths of stay by employing this technique of daily interruption of infusions. ${ }^{10,11}$ The largest RCT to test daily interruption of sedation was the SLEAP study $^{12}$ that randomized 430 patients in 16 Canadian and United States centres using protocolized sedation in all patients. In this trial, patients randomized to daily sedation interruption required more daily boluses and higher mean doses of opiates and benzodiazepines, and they did not achieve a reduction in the duration of mechanical ventilation or ICU length of stay. These results agreed with the inability of daily sedation interruption to reduce the total dose of sedation in each of the previous nonbeneficial trials. The authors of SLEAP suggested that the use of protocolized sedation in the control group may have been superior to the "usual care" in the previous RCTs that showed a benefit of daily awakening in earlier trials. They concluded that "The effectiveness of any new intervention to minimize sedation likely depends on the local usual care." ${ }^{12}$ Interestingly, in the current report ${ }^{6}$ (as stated above), only $22 \%$ of all sedative and opioid infusions were stopped and resumed as part of a planned daily interruption strategy (most were stopped in preparation for extubation or neurological assessment). This occurred despite the fact that these data were collected after the publication of the earlier trials suggesting that daily interruption was beneficial $^{7,9}$ but prior to the publication of the SLEAP trial $^{12}$ which showed no benefit.

Other strategies using protocols aimed at minimizing total dosages of sedation medication have also been shown to be helpful. A recently published Danish single-centre RCT in 140 patients ${ }^{13}$ focused on treating pain with opiates and delirium with antipsychotics and using sedation only if necessary. This approach decreased the duration of mechanical ventilation and ICU and hospital lengths of stay when compared with sedation infusions titrated to a Ramsey score of 3-4 combined with daily interruption. In addition, a single-centre Swiss RCT that randomized 137 patients to sedation titrated to a Ramsey score of 1-2 using boluses vs sedation titrated to a score of 3-4 using continuous infusions also showed a shorter duration of 
mechanical ventilation and ICU length of stay. ${ }^{4}$ Unlike these previous two trials, ${ }^{4,13}$ another single-centre Australian study enrolling 312 patients compared the use of a sedation protocol $v s$ local clinical practice and found no reduction in overall sedation doses and no improvements in duration of ventilation. ${ }^{14}$ These investigators suggested that customizing an intervention to individual patients based on acquired knowledge through education and the use of previous protocols could result in outperforming the inflexible application of an otherwise effective protocol. Indeed, effective protocols have been shown not to outperform nursing judgement in other ICU interventions. For example, in a recent crossover $\mathrm{RCT}^{15}$ nursing-directed glucose control in a cardiovascular ICU - where the importance of tight glucose control was accepted - was shown to be as effective and safe as using a validated insulin nomogram. ${ }^{16}$

In summary, patient benefit (duration of ventilation, ICU length of stay) was shown only in the trials that achieved total dose reductions as opposed to any particular strategy in the use of sedatives and opiate analgesics. This result suggests that clinicians should follow the principles of the most recent guidelines, i.e., employing an "analgesia first" strategy and targeting a sedation level as light as the patient on mechanical ventilation can tolerate because each component (regular assessment of pain, sedation, and delirium; treating pain and delirium; and targeting light sedation) will help reduce overall sedative use. The original trials which showed beneficial effects of daily interruption of sedation did, at that time, reduce overall use of sedation and challenge conventional thinking by showing that reduction could be achieved and patient outcomes could improve. As lighter sedation became more accepted by the critical care community, follow-up trials in which lighter sedation was more routinely applied have been unable to show a further reduction in sedation doses or clinical benefits by adding daily sedation interruption. Moreover, bolus dosing, if tolerated, appears to be the preferred approach except in very select cases (e.g., ongoing seizures, patient-ventilator dyssynchrony due to high ventilator settings to treat severe respiratory failure, intracranial hypertension, or in patients receiving neuromuscular blockade). If a sedative infusion can be stopped once, it likely should not be resumed; instead, it should be replaced with bolus dosing even if the patient is not yet ready for tracheal extubation. This will invariably lead to lower overall doses of sedation for the patient. It is less clear whether titration to sedation or pain scales provide added benefit. If the culture within the ICU fully supports the concept of minimal sedation, then specific titration algorithms may not be incrementally beneficial. Nevertheless, if there are residual concerns regarding potential patient discomfort or unplanned extubations, then titration protocols may help to reduce sedation levels. Notwithstanding these conclusions, we cannot simply assume that our sedation practices which do not follow protocols or interruption strategies are optimized; our practices should be continually re-examined and evaluated against the four principles identified above.

Regardless of the approach, it is clear from the comprehensive data provided by Burry et al. ${ }^{6}$ that almost all patients in ICUs receive sedatives, analgesics, and/or antipsychotic medications. Moreover, the RCT data clearly show that the manner in which ICU patients are treated with these medications has an impact on the quality of their experiences in the ICU as well as the duration of mechanical ventilation and ICU length of stay. Therefore, all critical care clinicians must continue to focus on sedation and analgesic practices to ensure they are providing optimal care for their patients.

\section{Optimiser l'approche de la douleur, de l'agitation et du délirium en unité de soins intensifs}

Les patients qui sont dans un état critique sous ventilation mécanique reçoivent pratiquement obligatoirement une analgésie et une sédation et, fréquemment, des antipsychotiques et des bloqueurs neuromusculaires. Ces interventions tentent de minimiser l'inconfort occasionné par le soutien physiologique invasif et d'améliorer la tolérance des patients et leur expérience aux soins intensifs en général. Semblable au concept de la «triade anesthésique » (analgésie, sédation et relâchement musculaire), le concept de «triade des soins intensifs » qui consiste à répondre à la douleur, l'agitation et au délirium, a été proposé pour les patients dans un état critique. ${ }^{1}$ Les recommandations les plus récentes publiées pour la gestion de la douleur, de l'agitation et du déirium en unité de soins intensifs (USI) sont les suivantes $\left.{ }^{2}: 1\right)$ évaluer régulièrement le niveau de douleur, de sédation et de délirium en utilisant des échelles validées; 2) donner la priorité au traitement de la douleur en utilisant des moyens à la fois non pharmacologiques et pharmacologiques; 3) donner la priorité aux moyens non pharmacologiques pour prévenir et traiter le délirium; et 4) utiliser des sédatifs pour maintenir des niveaux de sédation légers plutôt que profonds.

Cette stratégie vise à traiter plutôt qu'à masquer la maladie par l'utilisation de sédatifs seulement après avoir traité la douleur et le delirium. ${ }^{1}$ Bien que des cliniciens aient pu considérer par le passé qu'une sédation plus 
profonde était nécessaire pour améliorer la synchronisation patient-ventilateur (en particulier quand les ventilateurs d'USI avaient une moins grande capacité à se synchroniser avec les efforts respiratoires des patients) et pour minimiser le traumatisme psychologique perçu, les études récentes ont montré que le recours à une sédation plus légère n'étaient pas pires ${ }^{3}$ ou, en fait, diminuait les effets psychologiques indésirables. ${ }^{4,5}$ Les investigateurs travaillant dans cet important domaine d'étude recherchent donc la meilleure façon d'aborder cette triade d'USI, tout en minimisant les effets nuisibles éventuels des agents pharmacologiques utilisés et en optimisant les aboutissements importants pour les patients, telle que la réduction de la durée de la ventilation et de la durée de séjour en USI.

Ce mois-ci, dans le numéro du Journal, Burry et coll. ${ }^{6}$ rapportent les résultats d'une étude observationnelle prospective transversale menée dans des USI médicales et chirurgicales. Les auteurs ont enregistré des données pendant deux semaines sur l'utilisation des analgésiques, des sédatifs, des antipsychotiques et des bloqueurs neuromusculaires chez des patients consécutifs à partir de la mise en place d'une ventilation mécanique invasive. Les patients ont été suivis jusqu'à l'extubation trachéale, la trachéostomie, le décès ou un maximum de 30 jours. L'étude était axée sur l'utilisation de ces interventions, plutôt que sur leur impact sur les durées de ventilation ou de séjour. Les données ont été recueillies à partir d'un échantillon de commodité, quoique vaste, de 51 USI dans tout le Canada et les auteurs déclarent avoir cherché à constituer un échantillon varié incluant à la fois des hôpitaux universitaires et communautaires (supposant qu'ils devaient être en mesure de recueillir des données prospectives). Sept cent douze patients ont été recrutés entre février 2008 et avril 2009, soit cinq ans avant la publication de l'étude et avant la publication des lignes directrices les plus récentes. ${ }^{2}$ Presque tous les patients (92\%) ont reçu des analgésiques ou des sédatifs, principalement en perfusions continues: les opioïdes ont été utilisés le plus souvent (85\%), par rapport aux benzodiazépines $(62 \%)$ et au propofol rarement utilisé $(10 \%)$. Des antipsychotiques ont également été administrés à $10 \%$ des patients, presque exclusivement chez ceux dont les poumons étaient ventilés pendant plus de 48 heures. Des bloqueurs neuromusculaires ont été utilisés chez $18 \%$ des patients, ce qui constitue peut-être une proportion étonnamment élevée, considérant que les poumons des patients n'ont été ventilés que pour une durée médiane de seulement trois jours. D'un autre côté, seulement 58 des 130 patients ont reçu une dose d'agents bloquants neuromusculaires à plus d'une occasion si bien qu'il est difficile de savoir si ces doses uniques étaient liées à une procédure particulière ou avaient pour but d'aider la ventilation. Pour les patients dont les poumons ont été ventilés pendant sept jours ou plus, les opioïdes et les benzodiazépines ont été utilisés avec une égale fréquence (respectivement, 92,6\% contre 92,1\%). L'utilisation conjointe des opioïdes et des benzodiazépines a été associée de façon indépendante à une plus grande durée de la ventilation mécanique, à l'utilisation d'échelles d'analgésie ou de sédation et au recours à une contention physique. Des échelles de sédation, douleur et délirium ont été utilisées chez, respectivement, $53 \%, 19 \%$ et $5 \%$ des patients; en outre, $25 \%$ des analgésiques et/ou des sédatifs ont été titrés en suivant un protocole. Les protocoles de sédation étaient plus susceptibles d'être utilisés lorsque des échelles l'étaient aussi (ce qui a du sens puisque la plupart des protocoles impliquent une titration en fonction d'une échelle) chez des patients qui ont nécessité une ventilation prolongée et dans des hôpitaux communautaires plutôt que dans les hôpitaux universitaires. Les auteurs ont conclu «qu'en dépit des recommandations des sociétés professionnelles, l'utilisation de perfusions continues sans stratégies de sédation ciblée, comme dans un protocole, était fréquente. »

Burry et coll. ${ }^{6}$ ont également signalé que $22 \%$ de toutes les perfusions de sédatifs et d'opioïdes ont été interrompues et reprises dans le cadre d'une stratégie d'interruption quotidienne planifiée. Depuis la publication en 2000 de l'essai randomisé contrôlé de Kress et coll., ${ }^{7}$ l'interruption quotidienne de la sédation a amené la recherche dans ce domaine à considérer qu'il s'agissait d'une méthode permettant d'éviter de possibles effets indésirables de la sédation. Cette étude a constitué un tournant, impliquant 128 patients dans un état critique, randomisés pour bénéficier d'une interruption quotidienne de la sédation ou d'une interruption à la discrétion des cliniciens; elle a montré une diminution de l'utilisation des sédatifs et des analgésiques morphiniques, une diminution du nombre médian de jours sous ventilation mécanique de 2,4 jours (respectivement, 4,9 contre 7,3 jours; $P=0,004$ ), et une diminution de la durée médiane du séjour en USI de 3,5 jours (respectivement, 6,4 contre 9,9 jours; $P=0,02$ ). Dans cette étude unicentrique, les perfusions de sédation ont été titrées de façon à obtenir un score de 3 à 4 sur l'échelle de sédation de Ramsay qui classe le degré de sédation de 0 (patient agité ou sans repos) à 6 (patient endormi et ne répondant pas aux stimulations). ${ }^{8} \mathrm{Cet}$ essai clinique randomisé contrôlé a été suivi par un autre mené dans 4 centres et ayant inclus 336 patients dont l'interruption quotidienne des perfusions de sédation était combinée à une tentative quotidienne de respiration spontanée (l'étude d'éveil et de respiration contrôlés Awakening and Breathing Controlled) ${ }^{9}$ pour évaluer les possibilités d'extubation trachéale des patients. Le groupe bénéficiant de cette intervention a été comparé à un groupe témoin, également évalué quotidiennement pour la 
respiration spontanée; des échelles de sédation ont été utilisées. Cette étude a également montré des avantages comparables, mais contrairement à l'étude de référence, il y a eu significativement plus d'auto-extubations trachéales non prévues dans le groupe d'éveil quotidien (avec interruption des perfusions): respectivement, $10 \%$ contre $4 \%(P=0,03)$.

D'autres groupes d'investigateurs ont eu du mal à obtenir des diminutions des doses de sédatifs et les améliorations correspondantes sur la durée de l'hospitalisation en utilisant cette technique d'interruption quotidienne des perfusions. ${ }^{10,11} \mathrm{La}$ plus grande étude randomisée contrôlée ayant testé l'interruption quotidienne de la sédation était l'étude $\operatorname{SLEAP}^{12}$ qui a randomisé 430 patients dans 16 centres au Canada et aux États-Unis, en utilisant un protocole de sédation chez tous les patients. Dans cette étude, les patients randomisés dans le groupe d'interruption quotidienne de la sédation ont nécessité davantage de bolus quotidiens et des doses moyennes de morphiniques et de benzodiazépines plus importantes, sans obtenir de diminution de la durée de la ventilation mécanique ou du séjour en USI. Ces résultats concordaient avec le fait que l'interruption quotidienne de la sédation ne parvenait pas à faire baisser la dose totale de sédatifs dans chacune des études précédentes qui n'avaient pas montré les avantages de la méthode. Les auteurs de l'étude SLEAP ont suggéré que l'utilisation selon un protocole de la sédation dans le groupe contrôle avait pu être supérieure aux «soins habituels » des études randomisées contrôlées précédentes ayant montré un avantage du réveil quotidien. Leur conclusion était que «l'efficacité de n'importe quelle intervention destinée à minimiser la sédation dépend probablement des soins habituels locaux »..$^{12}$ Il est intéressant de noter que, dans la présente publication, ${ }^{6}$ seulement $22 \%$ de toutes les perfusions de sédatifs et d'opioïdes ont été arrêtées et reprises dans le cadre d'une stratégie planifiée d'interruption quotidienne (comme indiqué plus haut); la plupart des perfusions ont été arrêtées en préparation de l'extubation ou pour une évaluation neurologique. Cela a eu lieu bien que ces données aient été recueillies après la publication des études précédentes suggérant que l'interruption quotidienne était bénéfique, ${ }^{7,9}$ mais avant la publication de l'étude SLEAP $^{12}$ qui ne montrait aucun bénéfice.

D'autres stratégies utilisant des protocoles visant à minimiser les posologies totales de sédatifs se sont également avérées utiles. Une étude danoise unicentrique randomisée contrôlée ayant inclus 140 patients ${ }^{13}$ a porté principalement sur le traitement de la douleur par les morphiniques et du délirium par des antipsychotiques, en n'utilisant la sédation que si nécessaire. Cette approche a diminué la durée de la ventilation mécanique ainsi que les durées du séjour en USI et à l'hôpital quand on la comparait aux perfusions de sédation titrées pour atteindre un score de Ramsay de 3 à 4 associées à l'interruption quotidienne. De plus, une étude randomisée contrôlée unicentrique suisse qui avait randomisé 137 patients dans le groupe sédation titrée pour un score de Ramsey de 1 à 2 au moyen de bolus, contre une sédation titrée pour un score de 3 à 4 au moyen de perfusions continues, a également montré de plus courtes durées de ventilation mécanique et de séjour en USI. ${ }^{4}$ Contrairement à ces deux études precedents, ${ }^{4,13}$ une autre étude unicentrique australienne ayant inclus 312 patients a comparé l'utilisation d'un protocole de sédation et les pratiques cliniques locales: l'étude n'a trouvé aucune réduction globale des doses de sédatifs, ni de raccourcissement de la durée de la ventilation. $^{14}$ Ses investigateurs ont suggéré que la personnalisation d'une intervention pour chaque patient, basée sur les connaissances acquises par la formation et l'utilisation des protocoles précédents, pouvait entrainer de meilleurs résultats que l'application rigide d'un protocole par ailleurs efficace. De fait, il a été montré que des protocoles efficaces ne surpassent pas les traitements suivant l'opinion du personnel infirmier dans d'autres interventions en USI. Par exemple, dans une étude randomisée contrôlée récente avec croisement, ${ }^{15}$ le contrôle de la glycémie par les infirmières d'une USI cardiovasculaire (où l'importance d'un contrôle glycémique strict était admise) s'est avéré aussi efficace et sécuritaire que l'utilisation d'un nomogramme validé de l'insuline. ${ }^{16}$

En résumé, les avantages pour le patient (durée de la ventilation, durée du séjour en USI) n'ont été démontrés que dans les études ayant obtenu des réductions des doses totales, par opposition à toute autre stratégie particulière d'utilisation des sédatifs et des analgésiques morphiniques. Ce résultat suggère que les cliniciens doivent suivre les principes des lignes directrices les plus récentes, c'est-àdire, utiliser une stratégie « d'analgésie d'abord » et cibler un niveau de sédation la plus légère que le patient sous ventilation mécanique peut tolérer parce que chaque composante (évaluation régulière de la douleur, sédation et délire; traitement de la douleur et du délirium; et visée d'une sédation légère) contribuera à réduire l'utilisation globale des sédatifs. Les études originales qui ont montré les effets bénéfiques de l'interruption quotidienne de la sédation ont réduit, à ce moment-là, l'utilisation globale de la sédation et ont remis en cause les opinions conventionnelles, en montrant qu'une réduction était possible et que l'évolution des patients pouvait être améliorée. Au fur et à mesure que la sédation plus légère a été acceptée par la communauté professionnelle travaillant en soins intensifs, des études de suivi au cours de laquelle une sédation plus légère a été couramment 
utilisée n'ont pas réussi à montrer une diminution supplémentaire des doses de sédation ou des bénéfices cliniques par l'addition de l'interruption quotidienne de la sédation. De plus, l'administration de bolus, s'ils sont tolérés, semble être l'approche privilégiée sauf dans des cas très particuliers, notamment en cas de crises convulsives continues, mauvaise synchronisation patient-ventilateur (due aux réglages élevés du ventilateur pour le traitement d'une insuffisance respiratoire sévère), hypertension intracrânienne, ou chez des patients recevant un bloc neuromusculaire. Si une perfusion de sédation peut être arrêtée immédiatement, elle ne devrait probablement pas être reprise; elle devrait être plutôt remplacée par l'administration de bolus, même si le patient n'est pas encore prêt pour une extubation trachéale. Cela conduira invariablement à de plus faibles doses de sédation pour le patient. Il est plus difficile de dire si la titration nécessaire pour obtenir une sédation ou l'utilisation des échelles de douleur apporte un avantage supplémentaire. Si la culture régnant dans l'USI favorise le concept de sédation minimum, des algorithmes de titration spécifiques peuvent alors ne pas présenter de bénéfices supplémentaires. Néanmoins, s'il reste des préoccupations concernant un inconfort potentiel ressenti par le patient ou des extubations non planifiées, les protocoles de titrations peuvent contribuer à abaisser les niveaux de sédation. Malgré ces conclusions, il ne nous est pas permis de supposer que nos pratiques de sédation - qui ne suivent pas de protocoles - ou de stratégies d'interruption sont optimisées. Nos pratiques devraient être constamment réexaminées et évaluées contre les quatre principes identifiés plus haut.

Indépendamment de l'approche, il est clair à partir des données exhaustives fournies par Burry et coll. ${ }^{6}$ que presque tous les patients hospitalisés en USI reçoivent des sédatifs, des analgésiques et/ou des médicaments antipsychotiques. De plus, les données de l'étude clinique randomisée montrent clairement que la façon dont sont traités les patients en USI avec ces médicaments a un impact sur la qualité de leur expérience en USI, ainsi que sur la durée de la ventilation mécanique et de leur hospitalisation en soins intensifs. En conséquence, tous les cliniciens de soins intensifs doivent continuer à se concentrer sur les pratiques de sédation et de traitement de la douleur pour garantir la délivrance de soins optimaux à leurs patients.

\section{Conflicts of interest None declared.}

Conflits d'intérêts Aucun déclaré.

\section{References}

1. Reade MC, Finfer $S$. Sedation and delirium in the intensive care unit. N Engl J Med 2014; 370: 444-54.

2. Barr J, Fraser GL, Puntillo K, et al. Clinical practice guidelines for the management of pain, agitation, and delirium in adult patients in the intensive care unit. Crit Care Med 2013; 41: 263-306.

3. Strom T, Stylsvig M, Toft P. Long-term psychological effects of a no-sedation protocol in critically ill patients. Crit Care 2011; 15 : R293.

4. Treggiari MM, Romand JA, Yanez ND, et al. Randomized trial of light versus deep sedation on mental health after critical illness. Crit Care Med 2009; 37: 2527-34.

5. Kress JP, Gehlbach B, Lacy M, Pliskin N, Pohlman AS, Hall JB. The long-term psychological effects of daily sedative interruption on critically ill patients. Am J Respir Crit Care Med 2003; 168: 1457-61.

6. Burry LD, Williamson DR, Perreault MM, et al. Analgesic, sedative, antipsychotic, and neuromuscular blocker use in Canadian intensive care units: a prospective, multicentre, observational study. Can J Anesth 2014; 61: this issue. DOI: 10.1007/s12630-014-0174-1.

7. Kress JP, Pohlman AS, O'Connor MF, Hall JB. Daily interruption of sedative infusions in critically ill patients undergoing mechanical ventilation. N Engl J Med 2000; 342: 1471-7.

8. Ramsay MA, Savege TM, Simpson BR, Goodwin R. Controlled sedation with alphaxalone-alphadolone. BMJ 1974; 2: 656-9.

9. Girard TD, Kress JP, Fuchs BD, et al. Efficacy and safety of a paired sedation and ventilator weaning protocol for mechanically ventilated patients in intensive care (Awakening and Breathing Controlled trial): a randomised controlled trial. Lancet 2008; 371: 126-34.

10. Anifantaki S, Prinianakis G, Vitsaksaki E, et al. Daily interruption of sedative infusions in an adult medical-surgical intensive care unit: randomized controlled trial. J Adv Nurs 2009; 65: $1054-60$

11. de Wit M, Gennings C, Jenvey WI, Epstein SK. Randomized trial comparing daily interruption of sedation and nursingimplemented sedation algorithm in medical intensive care unit patients. Crit Care 2008; 12: R70.

12. Mehta S, Burry L, Cook D, et al; SLEAP Investigators; Canadian Critical Care Trials Group. Daily sedation interruption in mechanically ventilated critically ill patients cared for with a sedation protocol: a randomized controlled trial. JAMA 2012; 308: 1985-92.

13. Strom T, Martinussen T, Toft P. A protocol of no sedation for critically ill patients receiving mechanical ventilation: a randomised trial. Lancet 2010; 375: 475-80.

14. Bucknall TK, Manias E, Presneill JJ. A randomized trial of protocol-directed sedation management for mechanical ventilation in an Australian intensive care unit. Crit Care Med 2008; 36: 1444-50.

15. Chant C, Mustard M, Thorpe KE, Friedrich JO. Nurse- vs nomogram-directed glucose control in a cardiovascular intensive care unit. Am J Crit Care 2012; 21: 270-8.

16. Chant C, Wilson G, Friedrich JO. Validation of an insulin infusion nomogram for intensive glucose control in critically ill patients. Pharmacotherapy 2005; 25: 352-9. 